\title{
ON GROUPS WITH ALL SUBGROUPS SUBNORMAL OR SOLUBLE OF BOUNDED DERIVED LENGTH
}

\author{
KIVANÇ ERSOY \\ Department of Mathematics, Mimar Sinan Fine Arts University \\ İstanbul 34427, Turkey \\ e-mail: kivanc.ersoy@msgsu.edu.tr \\ ANTONIO TORTORA and MARIA TOTA \\ Dipartimento di Matematica, Università di Salerno \\ Via Giovanni Paolo II, 132 - Fisciano (SA) 84084, Italy \\ e-mail: antortora@unisa.it,mtota@unisa.it
}

(Received 28 July 2012; accepted 20 December 2012; first published online 13 August 2013)

\begin{abstract}
In this paper we deal with locally graded groups whose subgroups are either subnormal or soluble of bounded derived length, say $d$. In particular, we prove that every locally (soluble-by-finite) group with this property is either soluble or an extension of a soluble group of derived length at most $d$ by a finite group, which fits between a minimal simple group and its automorphism group. We also classify all the finite non-abelian simple groups whose proper subgroups are metabelian.
\end{abstract}

2010 Mathematics Subject Classification. 20F19, 20 E32.

1. Introduction. A well-known result, due to Möhres (see [12]), states that a group with all subgroups subnormal is soluble, while a result proved separately by Casolo [4] and Smith [16] shows that such a group is nilpotent if it is also torsion-free. Later, Smith [18] generalized these results to groups in which every subgroup is either subnormal or nilpotent. More precisely, he proved that a locally (soluble-by-finite) group with all subgroups subnormal or nilpotent is soluble, and the same holds for a locally graded group whose non-nilpotent subgroups are subnormal of bounded defect. Also, in both cases, the nilpotency follows if the group is torsion-free (see [17]). Recall that a group is locally graded if every non-trivial, finitely generated subgroup has a non-trivial finite image. The class of locally graded groups is rather wide and, in particular, it contains all locally (soluble-by-finite) groups. This restriction is made in order to avoid Tarski monsters (see [13]), which show that the previous results are false without any finiteness condition.

In this paper we are interested in studying locally graded groups with all subgroups subnormal or soluble. The first problem that arises here is the presence of finite minimal simple groups, i.e. non-abelian simple groups in which every proper subgroup is soluble. These have been completely classified by Thompson in [22]. Using this classification, in Section 2, we get all the finite non-abelian simple groups having each proper subgroup metabelian.

Another difficulty is due to infinite locally graded groups with all proper subgroups soluble. Such groups are both hyperabelian (see [8]) and locally soluble (see [7]), but it is still an open question whether these are soluble. However, there is a positive answer 
if we bound the derived length of subgroups (see [6]). Motivated by this result, we deal with locally graded groups whose subgroups are either subnormal or soluble of bounded derived length. In our analysis, almost minimal simple groups show up. These are groups which fit between a minimal simple group and its automorphism group.

In line with Smith's results $[\mathbf{1 7}, \mathbf{1 8}]$, our main theorems follow. These will be proved in Section 3.

THEOREM 1.1. Let $G$ be a locally (soluble-by-finite) group and suppose that, for some positive integer $d$, every subgroup of $G$ is either subnormal or soluble of derived length at most $d$. Then either

(i) $G$ is soluble, or

(ii) $G^{(r)}$ is finite for some integer $r$ and $G$ is an extension of a soluble group of derived length at most $d$ by a finite almost minimal simple group.

THEOREM 1.2. Let $G$ be a locally graded group and suppose that, for some positive integers $n$ and $d$, every subgroup of $G$ is either subnormal of defect at most $n$ or soluble of derived length at most $d$. Then either

(i) $G$ is soluble of derived length not exceeding a function depending on $n$ and $d$, or

(ii) $G^{(r)}$ is finite for some integer $r=r(n)$ and $G$ is an extension of a soluble group of derived length at most $d$ by a finite almost minimal simple group.

2. Minimal simple groups. In this section we focus on locally graded minimal simple groups. By [8, Lemma 2.4] such groups are necessarily finite and are known.

THEOREM 2.1 ([22], Corollary 1). Every finite minimal simple group is isomorphic to one of the following groups:

(i) $\operatorname{PSL}\left(2,2^{p}\right)$, where $p$ is any prime;

(ii) $\operatorname{PSL}\left(2,3^{p}\right)$, where $p$ is any odd prime;

(iii) $\operatorname{PSL}(2, p)$, where $p>3$ is any prime such that $p^{2}+1 \equiv 0(\bmod 5)$;

(iv) $\operatorname{PSL}(3,3)$;

(v) $\operatorname{Sz}\left(2^{p}\right)$, where $p$ is any odd prime.

The table below, which will be useful later, shows some of the details concerning the outer automorphism group of a finite minimal simple group $M$. By [5, p. xv], $|\operatorname{Out}(M)|=d \cdot f \cdot g$ where, $d$ is the order of the group of diagonal automorphisms, $f$ is the order of the group of field automorphisms and $g$ is the order of the group of graph automorphisms (modulo field automorphisms). For more details, see [5, Table 5, p. xvi].

Table 1. Outer automorphisms of a finite minimal simple group

\begin{tabular}{llllc}
\hline$M$ & $d$ & $f$ & $g$ & $|\operatorname{Out}(M)|$ \\
\hline $\operatorname{PSL}\left(2,2^{p}\right)$ & 1 & $p$ & 1 & $p$ \\
$\operatorname{PSL}\left(2,3^{p}\right), p \geq 3$ & 2 & $p$ & 1 & $2 p$ \\
$\operatorname{PSL}(2, p), p>3$ and $5 \mid\left(p^{2}+1\right)$ & 2 & 1 & 1 & 2 \\
$\operatorname{PSL}(3,3)$ & 1 & 1 & 2 & 2 \\
$\operatorname{Sz}\left(2^{p}\right), p \geq 3$ & 1 & $p$ & 1 & $p$ \\
\hline
\end{tabular}


In light of Theorem 2.1, we now classify all the finite non-abelian simple groups whose proper subgroups are metabelian.

Proposition 2.2. Let $G$ be a finite non-abelian simple group with every proper subgroup metabelian. Then $G$ is isomorphic to one of the following groups:

(i) $\operatorname{PSL}\left(2,2^{p}\right)$, where $p$ is any prime;

(ii) $\operatorname{PSL}\left(2,3^{p}\right)$, where $p$ is any odd prime;

(iii) $\operatorname{PSL}(2, p)$, where $p>3$ is any prime such that $p^{2}+1 \equiv 0(\bmod 5)$ and $p^{2}-1 \not \equiv 0$ $(\bmod 16)$.

Proof. It is enough to analyse each case of Theorem 2.1.

Let $q$ be a power of any prime. By [21, Theorem 6.25], $\operatorname{PSL}(2, q)$ contains a non-metabelian soluble subgroup if and only if it has a subgroup isomorphic to $S_{4}$, the symmetric group of degree 4. Also, by [21, Theorem 6.26], this is equivalent to the condition $q^{2} \equiv 1(\bmod 16)$. Hence, if $q=2^{p}$, then all subgroups of $\operatorname{PSL}\left(2,2^{p}\right)$ are metabelian. Suppose $q=3^{p}$, with $p=2 k+1$. Since $9^{2 k} \equiv 1(\bmod 16), S_{4}$ is never contained in $\operatorname{PSL}\left(2,3^{p}\right)$ and therefore all subgroups of $\operatorname{PSL}\left(2,3^{p}\right)$ are metabelian. Let $q=p>3$ with $p^{2}+1 \equiv 0(\bmod 5)$. If $p^{2}-1 \not \equiv 0(\bmod 16)$, all subgroups of $\operatorname{PSL}(2, p)$ are metabelian.

Now we have to consider $\operatorname{PSL}(3,3)$ and $S z\left(2^{p}\right), p \geq 3$. But $\operatorname{PSL}(3,3)$ has a subgroup isomorphic to $S L(2,3)$, which has derived length 3 ; so we finish with $S z(q)$, where $q=2^{p}$. By [20, Theorem 9], $S z(q)$ contains a Frobenius group $F$ of order $q^{2}(q-1)$. Moreover, $S z(q)$ has only one abelian subgroup of order dividing $q^{2}(q-1)$, that is cyclic of order $q-1$, and its normalizer is a dihedral group of order $2(q-1)$ (see [20], p. 137). Hence, $F$ is not metabelian.

REMARK 2.3. We can observe that every proper subgroup of a minimal simple group has derived length at most 5. By Theorem 2.1 and Proposition 2.2, we need to consider the following cases:

Let $G=P S L(2, p), p>3, p^{2}+1 \equiv 0(\bmod 5)$ and $p^{2}-1 \equiv 0(\bmod 16)$. Then by [21, Theorems 6.25, 6.26], $G$ has a subgroup isomorphic to $S_{4}$, which is soluble of derived length 3 . This is also a unique non-metabelian subgroup of $G$.

Let $G=P S L(3,3)$ and $H$ be a proper subgroup of $G$. Since $H$ is soluble, it contains a non-trivial normal elementary abelian subgroup. Thus, by [2, Theorem 7.1], one of the following holds:

(1) $H$ has a cyclic normal subgroup of index at most 3;

(2) $H$ has an abelian normal subgroup $K$ such that $H / K$ can be embedded into the symmetric group $S_{3}$;

(3) $H$ has a normal elementary abelian 3-subgroup $K$ such that $H / K$ can be embedded into $G L(2,3)$. Now the derived length of $G L(2,3)$ is 4 and so $H$ has derived length at most 5. Indeed, let $Z=Z(S L(3,3))$ and $H$ be the subgroup of $G$ given by

$$
\left\{\left(\begin{array}{lll}
a & b & c \\
d & e & f \\
0 & 0 & g
\end{array}\right) Z \mid a, b, c, d, e, f, g \in \mathbb{F}_{3}, \quad(a e-b d) g=1\right\} .
$$


Then

$$
K=\left\{\left(\begin{array}{ccc}
1 & 0 & c \\
0 & 1 & f \\
0 & 0 & 1
\end{array}\right) Z \mid c, f \in \mathbb{F}_{3}\right\}
$$

is an elementary abelian 3-subgroup of $H$ such that $H / K \cong G L(2,3)$.

Therefore, every proper subgroup of $\operatorname{PSL}(3,3)$ has derived length at most 5 and $\operatorname{PSL}(3,3)$ contains a subgroup of derived length 5 .

Let $G=S z\left(2^{p}\right)$ for $p \geq 3$. Then, by [23, Theorem 4.1], any maximal subgroup of $G$ has derived length at most 3 .

3. Main results. We start with some preliminary lemmas.

LEMMA 3.1. Let $H$ be a subgroup of a group $G$. If every subgroup containing $H$ is subnormal in $G$, then $G^{(r)} \leq H$ for some $r \geq 0$. In particular, $r=0$ if and only if $H=G$.

Proof. We may assume $H<G$. Then there exists a series from $H$ to $G$, and by [12, Theorem 7], each factor is soluble. Hence, we have an abelian series from $H$ to $G$, say $H=H_{0} \triangleleft H_{1} \triangleleft \ldots \triangleleft H_{r}=G$. As $G^{(i)} \leq H_{r-i}$, for all $i \geq 0$, we get $G^{(r)} \leq H$.

REMARK 3.2. In the previous lemma, if we also assume that the subgroups containing $H$ are subnormal in $G$ of defect at most $n \geq 1$, then by $[9,12.2 .8] r$ depends on $n$. See also [3].

LEMMA 3.3. Let $G$ be a locally graded group with all subgroups subnormal or soluble, and suppose that $N$ is a minimal non-soluble normal subgroup of $G$.

(i) If $N$ is infinite, then $G$ is hyperabelian.

(ii) If $N$ is finite, then $G$ is an extension of a soluble group by a finite almost minimal simple group.

Proof. Firstly, note that every subgroup of $G / N$ is subnormal so that $G / N$ is soluble by [12, Theorem 7].

(i) By [8, Lemma 2.4], $N$ is hyperabelian. Let $K=\left\langle K_{i}: K_{i}<N, K_{i} \triangleleft G\right\rangle$. If $K=N$, since each $K_{i}$ is soluble, $N$ has a $G$-invariant ascending abelian series. Hence, $G$ is hyperabelian, since $G / N$ is a soluble group. Now consider $K<N$ and assume for a contradiction that $G$ is not hyperabelian. Then $K$ is soluble and so by [10, Corollary] $G / K$ is locally graded. Moreover, $G / K$ is not hyperabelian and its normal subgroup $N / K$ is minimal non-soluble. However, $N / K$ is hyperabelian and thus $N / K$ is also infinite. We can therefore restrict to the case $K=1$.

Let $A$ be a non-trivial normal abelian subgroup of $N$. Then $N=A^{G}$ so that $N$ is a product of normal abelian subgroups. This implies that $N$ is locally nilpotent. If $T$ is its torsion subgroup, we have either $T=1$ or $T=N$. Let $T=1$. As solubility is a countably recognizable property, we have that $N$ is countable. It is also locally nilpotent and torsion-free. Then by [11, Lemma 2], there exists $M<N$ such that the isolator $I_{N}(M)$ equals $N$. Also, $I_{N}(M)^{(i)} \leq I_{N}\left(M^{(i)}\right.$ ) for all $i \geq 0$ (see, for instance, $[9,2.3 .9])$. As $M$ is soluble, so is $N$, a contradiction. Assume $T=N$. Then $N$ is a locally finite $p$-group. Clearly $Z(N)=1$ and so we may apply [1, Lemma 2.1] to $N$. It follows that there exists $m>0$ such that $R=\langle Z(H): H \triangleleft N, d(H)>m\rangle$ is a proper 
subgroup of $N$, where $d(H)$ denotes the derived length of $H$. On the other hand, $N$ has a finitely generated soluble subgroup of derived length greater than $m$. This means that there is a subgroup $L$ of $N$ generated by finitely many abelian normal subgroups that is necessarily nilpotent but of derived length $>m$. The set $J$ of all such subgroups $L$ is invariant under $\operatorname{Aut}(N)$. So the subgroup $\bar{R}=\langle Z(L): L \in J\rangle$ is characteristic in $N$ and normal in $G$. Furthermore, $\bar{R} \leq R<N$. Thus, $\bar{R}=1$ and this is a contradiction.

(ii) Let $S$ be the soluble radical of $N$. Surely, $S$ is a characteristic subgroup of $N$ and so it is normal in $G$. Without loss of generality, assume $S=1$. Then $N$ is a finite non-abelian simple group, hence $C_{G}(N) \cap N$ is trivial. This gives that $C_{G}(N)$ is soluble. Since $G / C_{G}(N)$ embeds in $\operatorname{Aut}(N)$, we get that $G$ is soluble-by-finite. This also implies that the soluble radical of $G$ is soluble. Suppose it is trivial so that $G$ is finite.

Let $M$ be a minimal normal subgroup of $G$. Then $M$ is a direct product of copies of a non-abelian simple group $A$. Obviously, $A$ is subnormal in $G$ and so, by Lemma 3.1, we have $G^{(r)} \leq A$ for some $r>0$. Thus, $A=M$. It follows that $M$ is a minimal simple group and, by Möhres' result (see [12] or [9, 12.2.1]), $G / M$ is soluble. Moreover, $C_{G}(M) \cap M=1$ so that $C_{G}(M)$ is a normal soluble subgroup of $G$. By our assumption, $C_{G}(M)=1$ and this gives $G \lesssim A u t(M)$.

LEMMA 3.4. Let $G$ be a locally (soluble-by-finite) group with all subgroups subnormal or soluble. Then either

(i) $G$ is locally soluble, or

(ii) $G^{(r)}$ is finite for some integer $r$ and $G$ is an extension of a soluble group by a finite almost minimal simple group.

Proof. Let $K=G^{(\alpha)}=G^{(\alpha+1)}$, a perfect group. If $K=1$, then $G$ has a descending normal series with abelian factors. Since $G$ is locally (soluble-by-finite), it follows that $G$ is locally soluble. Suppose $K \neq 1$, and let $H$ be a proper subgroup of $K$ that is not soluble. Then all subgroups containing $H$ are subnormal in $G$ and, by Lemma 3.1, there is an integer $r$ such that $G^{(r)} \leq H$, a contradiction. Thus, every proper subgroup of $K$ is soluble and $K$, being perfect, is a minimal non-soluble group. Furthermore, $G / K$ is soluble so that $\alpha$ is finite. The claim is now a consequence of Lemma 3.3.

Note that, proving ( $i$ ) of Lemma 3.4, we have that the derived series of $G$ ends in finitely many steps. The next lemma, which follows from [19, Proposition 1] together with $[9,12.2 .6]$, shows that this also happens when $G$ is locally soluble.

LEMMA 3.5. Let $\mathfrak{X}=\bigcup_{i \in \mathbb{N}} \mathfrak{X}_{i}$ be a class of groups where each class $\mathfrak{X}_{i}$ is closed under taking subgroups and direct limits, and $\mathfrak{X}_{i} \subseteq \mathfrak{X}_{i+1}$ for all $i$. Let $G$ be a group with all subgroups subnormal or in $\mathfrak{X}$, and suppose that $G \notin \mathfrak{X}$. If $G$ is locally soluble, then $G^{(r)}=G^{(r+1)}$ for some integer $r$.

Now we can prove Theorem 1.1.

Proof of Theorem 1.1. By Lemma 3.4, $G$ is either locally soluble, or $G^{(r)}$ is finite for some integer $r$ and $G$ is an extension of a soluble group $S$ by a finite almost minimal simple group. If $S$ is not soluble of derived length at most $d$ then, by Lemma 3.1, $G^{(r)} \leq S$ and $G$ is soluble. Let $G$ be locally soluble and suppose that it is not soluble. By Lemma 3.5 with $\mathfrak{X}$ the class of soluble groups, we have $G^{(s)}=G^{(s+1)}$ for some $s \geq 0$. Moreover, $G^{(s)}$ is not soluble. It follows, as in the proof of Lemma 3.4, that every proper subgroup of $G^{(s)}$ is soluble of length at most $d$. Thus, $G^{(s)}$ is finite by [6, Lemma 2.1], a contradiction. 
According to a theorem by Roseblade (see [15] or [9, 12.2.3]), a group in which every subgroup is subnormal of defect at most $n \geq 1$ is nilpotent of class not exceeding a function depending only on $n$. Using this, we can generalize Lemma 3.4 to the locally graded case, provided that the subnormal defect is bounded.

LEMMA 3.6. Let $G$ be a locally graded group and suppose that, for some positive integer $n$, every non-soluble subgroup of $G$ is subnormal of defect at most $n$. Then $G$ is locally (soluble-by-finite).

Proof. We may assume that $G$ is finitely generated. Suppose that it is not soluble-byfinite and denote by $R$ its finite residual. As $G$ is locally graded, $R$ is a proper subgroup of $G$. Let $N$ be a normal subgroup of $G$ with finite index. Then every subgroup of $G / N$ is subnormal of defect $\leq n$ and so, by Roseblade's theorem [9, 12.2.3], $G / N$ is nilpotent of bounded class depending on $n$. It follows that $G / R$ is nilpotent and $R$ is not soluble. Let $S$ be a proper subgroup of $R$ and suppose that $S$ is not soluble. Then every subgroup of $R / S^{R}$ is subnormal of defect $\leq n$, in particular $R / S^{R}$ is soluble, by [9, 12.2.3]. This implies that $R^{\prime}<R$. So $G / R^{\prime}$ is finitely generated and abelian-by-nilpotent. We get that $G / R^{\prime}$ is residually finite (see, for instance, [14, Theorem 9.51]) and $R^{\prime}=R$, a contradiction. Hence, every proper subgroup of $R$ is soluble and $R$ cannot be finite, otherwise $G$ would be finite-by-nilpotent and, consequently, also nilpotent-by-finite. By Lemma 3.3, we obtain that $G$ is hyperabelian. Then $G$ has a finite non-nilpotent image $G / M$ (see, for instance, [14, Theorem 10.51]). If $M$ is soluble then $G$ is solubleby-finite, so $M$ is not soluble and every subgroup of $G / M$ is subnormal of defect $\leq n$. Thus, $G / M$ is nilpotent by $[9,12.2 .3]$, a contradiction.

Proof of Theorem 1.2. By Lemma 3.6, jointly with Theorem 1.1, we have that $G$ is either soluble or $G^{(r)}$ is finite for some $r \geq 0$ and $G$ is an extension of a soluble group of derived length at most $d$ by a finite almost minimal simple group. Let $G$ be soluble, and denote by $e$ its derived length. We may assume $d<e$. Then $H=G^{(e-(d+1))}$ is soluble of length $d+1$ and every subgroup of $G$ containing $H$ is subnormal of defect $\leq n$. It follows that $G^{(s)} \leq H$ for some $s$ depending on $n$ (see Remark 3.2). Thus, $G$ is soluble of length at most $s+d+1$. Suppose now that there exists $r \geq 0$ such that $K=G^{(r)}$ is finite and non-soluble. Since every subgroup of $G$ containing $K$ is subnormal of defect $\leq n$, we get, as before, $G^{(t)} \leq K$ for some $t$ depending on $n$.

As a final remark we point out that in (ii) of Theorems 1.1 and 1.2, one cannot expect that $G$ is an extension of a soluble group $S$ by a finite minimal simple group: it suffices to consider the direct product of any abelian group by the symmetric group of degree 5. However, if $M / S$ is a finite minimal simple subgroup of $G / S$ such that $G / S \lesssim \operatorname{Aut}(M / S)$, then $M \triangleleft G$ by Lemma 3.1 and we can compute the order of $G / M$. In fact, $G / M \lesssim \operatorname{Out}(M / S)$, where $|\operatorname{Out}(M / S)|$ divides $2 p$, with $p$ odd prime, by Table 1 .

AcKnowledgements. The authors would like to thank Prof. Howard Smith for interesting discussions and useful suggestions. This study was carried out during the first author's visit to the University of Salerno and is supported by The Scientific and Technological Research Council of Turkey (TÜBİTAK) BİDEB 2219 International Post Doctoral Research Fellowship. The first author thanks TÜBİTAK for the support. 


\section{REFERENCES}

1. A. Arikan, S. Sezer and H. Smith, On locally finite minimal non-solvable groups, Cent. Eur. J. Math. 8 (2010), 266-273. $150-178$.

2. D. M. Bloom, The subgroups of PSL(3, q) for odd $q$, Trans. Am. Math. Soc. 127 (1967),

3. C. Casolo, Groups in which all subgroups are subnormal, Rend. Accad. Naz. Sci. XL Mem. Mat. 10(5) (1986), 247-249.

4. C. Casolo, Torsion-free groups in which every subgroup is subnormal, Rend. Circ. Mat. Palermo 50(2) (2001), 321-324.

5. J. H. Conway, R. T. Curtis, S. P. Norton, R. A. Parker and R. A. Wilson, Atlas of finite groups (Oxford University Press, Oxford, UK, 1985).

6. M. R. Dixon and M. J. Evans, Groups with the minimum condition on insoluble subgroups, Arch. Math. 72 (1999), 241-251.

7. M. R. Dixon, M. J. Evans and H. Smith Groups with proper subgroups of certain types, Ischia Group Theory 2006 (World Scientific Publishing, Hackensack, NJ, 2007), 73-82.

8. S. Franciosi, F. de Giovanni and M. L. Newell, Groups with polycyclic non-normal subgroups, Algebra Colloq. 7 (2000), 33-42.

9. J.C. Lennox and D. J.S. Robinson, The theory of infinite soluble groups (Oxford University Press, Oxford, UK, 2004).

10. P. Longobardi, M. Maj and H. Smith, A note on locally graded groups, Rend. Sem. Mat. Univ. Padova 94 (1995), 275-277.

11. W. Möhres, Torsionsfreie Gruppen, deren Untergruppen alle subnormal sind, Math. Ann. 284 (1989), 245-249.

12. W. Möhres, Auflösbarkeit von Gruppen, deren Untergruppen alle subnormal sind, Arch. Math. 54 (1990), 232-235.

13. A. Yu. Olshanskii, Groups of bounded period with subgroups of prime order, Algebra Logic 21 (1982), 369-418.

14. D. J. S. Robinson, Finiteness conditions and generalized soluble groups, Part 1 and Part 2 (Springer-Verlag, Berlin, Germany, 1972).

15. J. E. Roseblade, On groups in which every subgroup is subnormal, J. Algebra 2 (1965), $402-412$. $1-6$.

16. H. Smith, Torsion-free groups with all subgroups subnormal, Arch. Math. 76 (2001),

17. H. Smith, Torsion-free groups with all non-nilpotent subgroups subnormal, Topics in Infinite Groups, 297-308, Quad. Mat. 8 (Dept. Math., Seconda Univ. Napoli, Caserta, 2001).

18. H. Smith, Groups with all non-nilpotent subgroups subnormal, topics in infinite groups, Quaderni di Matematica, 8 (2001), 309-326 (Dept. of Math., Seconda Univ. Napoli, Caserta).

19. H. Smith, Groups with all subgroups subnormal or nilpotent-by-Chernikov, Rend. Sem. Mat. Univ. Padova 126 (2011), 245-253.

20. M. Suzuki, On a class of doubly transitive groups, Ann. Math. 75 (1962), 105-145.

21. M. Suzuki, Group theory I (Springer-Verlag, Berlin, Germany, 1982).

22. J. G. Thompson, Nonsolvable finite groups all of whose local subgroups are solvable, Bull. Amer. Math. Soc. 74 (1968), 383-437.

23. R. A. Wilson, The finite simple groups, Graduate Texts in Mathematics, No. 251 (Springer-Verlag, London, 2009). 